\title{
Compromiso auditivo en osteogénesis imperfecta
}

\author{
Hearing compromise in imperfect osteogenesis
}

\author{
Michel Royer $F^{1}$, Carlos Stott $C^{1}$, Carolina Lara $F^{2}$, Lenka Franulic $C^{2}$.
}

\section{INTRODUCCIÓN}

La osteogénesis imperfecta es una enfermedad genética que se produce por un defecto en la síntesis de las cadenas alfa 1 y alfa 2 del colágeno tipo 1, debido a una alteración en los genes CL1A1 y COL1A2, localizados en los cromosomas 7 y 17. Tiene una variada presentación clínica, desde la letalidad en el período neonatal hasta un diagnóstico tardío en edad adulta, pudiendo comprometer cualquier sistema. Sus características más significativas son la fragilidad ósea y la coloración azul de las escleras. Su prevalencia estimada es de 1 en 10 mil habitantes, generalmente como una forma heredada en patrón dominante'.

La osteogénesis imperfecta se ha clasificado clásicamente, según Sillence ${ }^{2}$, en cuatro tipos: a). Tipo I: el más frecuente, de herencia autosómica dominante, caracterizado por fragilidad ósea leve, fracturas óseas tardías, escleras azules y compromiso auditivo en aproximadamente el $50 \%$ de los casos; b) Tipo II: es la forma más severa, de herencia autosómica recesiva, y determina una extrema fragilidad ósea con fracturas perinatales, escleras azules y curso letal; c) Tipo III: es el más severo entre quienes sobreviven al período neonatal. Presenta herencia autosómica recesiva o dominante, con fracturas severas, deformidad ósea, escleras normales y una asociación menor con hipoacusia; y d) Tipo IV: es de herencia autosómica dominante, con estatura baja, huesos frágiles, escleras normales y, algunas veces, compromiso auditivo. Posteriormente se han agregado tres nuevos tipos sin una caracterización completa ${ }^{3}$.

\section{COMPROMISO AUDITIVO}

La tríada clásica de esclera azul, fracturas espontáneas y compromiso auditivo fue descrito, inicialmente, por Van der Hoeve y De Keyn en $1917^{4}$. 日 compromiso auditivo puede ser de conducción, sensorioneural o mixto. Su frecuencia fluctúa entre $26 \%$ y $78 \%$, estimándose que se presenta en $50 \%$ en mayores de 50 años $^{5}$. Si bien la hipoacusia se instala habitualmente alrededor de la segunda dé cada de la vida, ésta ha sido detectada en un porcentaje variable de niños con osteogénesis imperfecta, por lo cual es altamente recomendable realizar un examen de pesquisa a los diez años y controlar la evolución 6,7 .

日 componente de conducción se tiende a presentar en la segunda década de la vida, por fijación de la platina del estribo y, a veces, por fracturas de las cruras de éste ${ }^{8}$. Hasta la década de los setenta se interpretaba la otoesclerosis como

\footnotetext{
${ }^{1}$ Médico del Servicio de Otorrinolaringología, Hospital Cínico de la Universidad de Chile.

${ }^{2}$ Interna Escuela de Medicina, Hospital Cínico de la Universidad de Chile.
} 
una manifestación localizada de esta enfermedad. Sin embargo, en la actualidad, se asume que el compromiso en la osteogénesis imperfecta es una manifestación local de un desorden esquelético generalizado, completamente diferente de la otoesclerosis, con una histología y un patrón enzimático distintos. Entre las características clínicas destaca un inicio más precoz, mayor compromiso del oído medio y compromiso sensorioneural más frecuente ${ }^{9}$. No obstante, será la investigación genética la que permitirá en definitiva separar ambas entidades.

日 componente sensorioneural de la hipoacusia en la osteogénesis imperfecta tiende a aparecer en la tercera década de la vida. No obstante, la pérdida en las frecuencias agudas es un hallazgo común, independiente de la edad, y es el resultado de microfracturas, hemorragias y/o invasión de la cóclea por tejido fibrovascular ${ }^{10,11}$.

Un factor a considerar en el diagnóstico y tratamiento de estos pacientes es la progresión de la hipoacusia $\mathrm{日}$ promedio de incremento anual del compromiso sensorioneural es de $0,6 \mathrm{~dB}$ en $500 \mathrm{~Hz}$ y $1,3 \mathrm{~dB}$ en $8 \mathrm{kHz}$, mientras que en el componente conductivo este incremento es constante de 0,4 dB para todas las frecuencias. Esta progresión ocurre en todos los pacientes con compromiso auditivo, sin influencia de eventos quirúrgicos, correspondiendo a la evolución natural de la enfermedad ${ }^{12}$.

\section{HALLAZGOS ANATÓMICOS}

La osteogénesis imperfecta se asocia, al menos en las formas clínicas con hipoacusia significativa, con alteraciones anatómicas e histológicas del oído. Sus características histológicas son: extensos remanentes de cartílago en el hueso encondral, retardo en la osificación de la capa encondral y periostal, finas y frágiles trabéculas, cruras frágiles del estribo y alteraciones óseas en los tres huesecillos ${ }^{13,14}$. Debido a estas alteraciones, es posible constatar durante la intervención quirúrgica, fracturas espontáneas de las cruras, y aún cuando éstas no estén presentes se debe actuar con sumo cuidado en la manipulación del estribo.
Con relación a la platina del estribo, ésta clásicamente se describe con un mayor grosor al habitual, existiendo una alta frecuencia de platina obliterada, $27 \%$ a $80 \%$, con una incidencia promedio de $51 \%{ }^{15,16}$. Esto es importante para el cirujano otólogo, puesto que la severidad del compromiso de la platina se relaciona con una mayor tasa de fracaso quirúrgico ${ }^{16}$. La mucosa del oído medio se encuentra hiperplásica e hiperémica, como resultado de la hipervascularización del promontorio; esto puede provocar problemas con el sangrado durante la cirugía, lo que se describe entre el $21 \%$ y $50 \%$ de las intervenciones ${ }^{17}$. También se han reportado fracturas espontáneas de cruras, obliteración de la ventana redonda y alteraciones en yunque y martillo 8 .

\section{TRATAMIENTO}

日 tratamiento del compromiso auditivo en esta enfermedad se basa en optimizar la audición del paciente sin generar un riesgo mayor. De esta forma se ha utilizado audífonos como una alternativa altamente efectiva pero, tal como ocurre en la otoesclerosis, existe un porcentaje de pacientes que no logra plena satisfacción con su uso. Además, los portadores de hipoacusia mixta en la que el audífono no es completamente útil, pudieran optar por la cirugía para alcanzar un nivel óptimo para el uso de audífonos.

Hasta la década de los '70 la cirugía del estribo en osteogénesis imperfecta se consideraba riesgosa ${ }^{18}$, debido a las alteraciones anatómicas del oído medio descritas previamente. Sin embargo, desde entonces varios autores han publicado resultados satisfactorios en pacientes con esta condición ${ }^{15,19-21}$. En recientes series realizando estapedostomías con prótesis de teflón se ha alcanzado un éxito sobre el $75 \%$ de los $\operatorname{casos}^{8,22}$, mientras que la incidencia de compromiso sensorioneural posoperatorio se encuentra entre el $1,7 \%$ y el $8 \%{ }^{19,21}$. Actualmente existe consenso respecto a que esta cirugía del estribo no es tan exitosa como en pacientes con otoesclerosis, mas no constituye una contraindicación para su realiza- 
ción. Una interesante experiencia publicada es un seguimiento nacional finlandés en el cual los resultados auditivos posquirúrgicos fueron peores ${ }^{23}$. Empero, debe considerarse que en éste participaron varios cirujanos, por lo que no sería comparable con estudios unipersonales. Además, en esta misma investigación se destaca el hecho que tanto hospitales universitarios como cirujanos con mayor experiencia lograron mejores resultados que el resto de los centros del país. Este punto enfatiza la importancia de resolver este tipo de patología con los mejores cirujanos, que tengan vasta experiencia en otoesclerosis y en centros donde se intervenga frecuentemente a estos pacientes.

$\mathrm{\theta}$ resultado en los seguimientos de pacientes operados demuestra una estabilidad del efecto quirúrgico en cuanto al cierre de la diferencia óseoaérea $^{22}$. No obstante, debe recordarse que el efectuar exitosamente esta cirugía no evitará la progresión sensorioneural, por lo que la terapia quirúrgica no determina el fin del compromiso auditivo, pero sí constituye una alternativa efectiva al uso de audífonos.

En series de pacientes en las cuales se ha determinado las causas del fracaso de la cirugía destaca la presencia de cambios obliterativos en la platina ${ }^{24}$, alteraciones en la mucosa del oído medio con importante sangrado intraoperatorio, y alteraciones en la cadena osicular que han debido ser resueltas con maleo-vestíbulopexia ${ }^{25}$. Frente a algunos de estos inconvenientes, el uso del láser ha demostrado facilitar la cirugía del estribo.

Aro hecho a destacar es que dos pacientes que tuvieron pérdida sensorioneural posquirúrgica, eran portadores de osteogénesis imperfecta tipo III, presentando severas alteraciones en el oído medio, con anomalías en el trayecto del nervio facial y la cadena osicular23,25. Este hecho desaconsejaría la cirugía en este tipo de pacientes.

En un número pequeño de pacientes con osteogénesis imperfecta y compromiso sensorioneural profundo se ha utilizado implantes cocleares. Si bien dentro de los hallazgos quirúrgicos destaca la hipervascularización de la mucosa, éstos han logrado ser efectuados exitosamente y demuestran ser altamente efectivos, tanto como en otras indicaciones de implante coclear ${ }^{26-29}$. Entre los escasos casos reportados ha existido estimulación no acústica, probablemente por una menor impedancia al flujo eléctrico debido a la densidad ósea anormal, pero ésta ha podido ser solucionada ajustando la programación ${ }^{26,28}$.

En resumen, es frecuente encontrar compromiso auditivo en los pacientes con osteogénesis imperfecta $y$, actualmente nuestra especialidad cuenta con eficaces alternativas de tratamiento. Ajustar éstas según la condición del paciente y la experiencia de los equipos quirúrgicos pareciera ser la alternativa más apropiada pero, debido a que no existen reportes al respecto en nuestro país, esta carencia nos debiera impulsar a revisar nuestras propias series para adoptar las conductas más apropiadas.

\section{BIBLIOGRAFÍA}

1. Byers P. Osteogenesis imperfecta. En: RoyceP, Steinman B. Connective Tissue and its Heritable Disorders: Molecular, Genetic and Medical Aspects. New York, NY: Wiley-Liss, 1993: 317350.

2. Sillence DO, Senn A, Danks DM. Genetic heterogeneity in osteogenesis imperfecta. $J$ Med Genet 1979; 16: 101-16.

3. Rauch F, GoorieuX F. Osteogenesis imperfecta. Lancet 2004;363:1377-85.

4. Van der hoeve J, De Klen A. Baluwe sclera, brooished van het beenstelsel. Ned Tijdschr Geneeskd 1917; 61: 1003-10.

5. Patrason CR, Monk EA, Mc Allion SJ. How common is hearing impairment in osteogenesis imperfecta? J Laryngol Otol 2001; 115: 280-2.

6. ImanI P, VIJAYASekaran S, LaNnigan $F$. Is it necessary to screen for hearing loss in the paediatric population with osteogenesis imperfecta? Cin Atolaryngol Allied Sci 2003; 28: 199-202.

7. KUURILA K, GRENMAN R, JOHANSSON R, KAITLA I. Hearing loss in children with osteogenesis imperfecta. Eur J Pediatr 2000; 159: 515-9. 
8. AlbaHnASAMy L, KISHOREA, ORelly BF. Results of stapes surgery on patients with osteogenesis imperfecta. Cin Otolaryngol Allied Sci 2001; 26: 473-6.

9. Holdsworth CE, EgNDAHL GL, SOI円R N, RICHARDSON KE, EYRING EJ. Comparative biochemical study of otosclerosis and osteogenesis imperfecta. Arch Otolaryngol 1973; 98: 336-9.

10. SHAPIRO J, PIKUS A, WeSS G, ROwE DW. Hearing and middle ear function in osteogenesis imperfecta. JAMA 1982; 247: 2120- 6.

11. ALTMAN F, KORNRID M. Osteogenesis imperfecta and otosclerosis: New investigations. Ann Otol Rhinol Laryngol 1967; 76: 89-104.

12. Garretsen AJ, Oremers CW, Huygen PL. Hearing loss (in nonoperated ears) in relation to age in osteogenesis imperfecta type I. Ann Otol Rhinol Laryngol 1997; 106: 575-82.

13. SCHUKNECHT H. Disorders of Bone. En: Schuknecht $\mathrm{H}$. Pathology of the ear. Philadelphia: Lea \& Febiger, 1993: 365-411.

14. NAGER GT. Fibrous dysplasia. En: Pathology of the Temporal Bone. Baltimore: Williams \& Wilkins, 1993: 1082-148.

15. Ferekidis E, StaVROUlakI P, Vossinakis I et al. Stapedotomy in osteogenesis imperfecta patients. J Laryngol Otol 2000; 114: 424-8.

16. GaRRetsen AJ, CReMers CW. Stapes surgery in osteogenesis imperfecta: Analysis of postoperative hearing loss. Ann Otol Rhinol Laryngol 1991; 100:120-30.

17. Die日g R, MüLlø J, HeMS J. Stapes surgery in osteogenesis imperfecta patients. Eur Arch Otorhinolaryngol 1997; 254: 120-7.

18. RIEDNER ĐD, LEVIN LS, HOLLIDAY MJ. Hearing patterns in dominant osteogenesis imperfecta. Arch Oolaryngol 1980; 106: 737-40.

19. SHEA JJ, POSTMA DS. Findings and long-term results in the hearing loss of osteogenesis imperfecta. Arch Aolaryngol 1982; 108: 467-70.
20. Pegersen U. Osteogenesis imperfecta clinical features, hearing loss and stapedectomy. Biochemical, osteodensitometric, cornometric and histologic aspects in comparison with otosclerosis (thesis). Acta Otolaryngol 1985; 415 (suppl): 1-36.

21. GarRetsen TJ, CRemers CW. Ear surgery in osteogenesis imperfecta Clinical findings and short-term and long-term results. Arch Aolaryngol Head Neck Surg 1990; 116: 317-23.

22. GOLABEK W, SZYMANSKI M, SIMIEC H. Results of stapedectomy in osteogenesis imperfecta. Otolaryngol Pol 2002; 56 (3): 323-6.

23. KUURILA K, PYNnÖNen S, GRÉMan R. Stapes surgery in osteogenesis imperfecta in Finland. Ann Otol Rhinol Laryngol 2004; 113: 187-93.

24. VInCent R, GRATACAP B, OATES J, Sperling N. Stapedotomy in osteogenesis imperfecta: $A$ prospective study of 23 consecutive cases. Otol Neurotol 2005; 26 859-65.

25. VAN DER RIJT A, CREMERS C. Stapes surgery in osteogenesis imperfecta: Results of a new se ries. Otol Neurotol 2003; 24: 717-22.

26. SZILVASSY J, JORI J, CZIGNER J, TOTH F, SZILVASSY Z, KISS JG Cochlear implantation in osteogenesis imperfecta. Acta Otorhinolaryngol Belg 1998; 52: 253-6.

27. HUANG TS, YeN PT, LIU SY. Cochlear implantation in a patient with osteogenesis imperfecta and otospongiosis. Am J Otolaryngol 1998; 19: 209-12.

28. STREuB日 SO, LUSTING LR. Cochlear implantation in patients with osteogenesis imperfecta. Aolaryngol Head Neck Surg 2005; 132: 73540.

29. Migirov L, HeNKIN Y, HiLdeSHemer M, KRONENBERG J. Cochlear implantation in a child with osteogenesis imperfecta. Int $J$ Pediatr Aorhinolaryngol 2003; 67: 677-80.

Dirección: Dr. Michel Royer Faúndez

Av. Santos Dumont 999. Independencia

Fono-Fax: 7777338

Email: nazorej@yahoo.com 